\title{
Utilidad del ecodoppler de vasos supraaórticos para el diagnóstico del síndrome de robo subclavio coronario
}

\section{Utility of supra-aortic doppler ultrasound for the diagnosis of coronary subclavian steal syndrome}

\author{
Martín Fernández ${ }^{*}$, Federico Cardone ${ }^{1}$, Martín Lombardero ${ }^{2}$ y Hugo Grancelli ${ }^{3}$ \\ ${ }^{1}$ Unidad Coronaria y Sección de Imágenes Cardiovasculares; ${ }^{2}$ Unidad de Imágenes Cardiovasculares; ${ }^{3}$ Servicio de Cardiología. Sanatorio Trinidad \\ Palermo. Buenos Aires, Argentina
}

\section{Introducción}

En los pacientes con antecedente de operación de revascularización miocárdica (ORM) con puente de arteria mamaria interna (AMI), la estenosis significativa $(>75 \%)$ o la oclusión de la arteria subclavia (AS) homolateral proximal pueden provocar disminución del flujo hacia el puente e incluso revertir el flujo en el mismo e inducir signos de isquemia miocárdica y constituir así el síndrome de robo subclavio-coronario (SRSC) $)^{1,2}$. Se presenta el caso de una paciente con extensa enfermedad vascular periférica y antecedente de ORM que evoluciona con dicho síndrome en el posoperatorio de una operación vascular periférica.

\section{Caso clínico}

Paciente femenina de 74 años de edad, hipertensa, con tabaquismo activo y antecedente de infarto de miocardio seis años antes que requirió ORM con tres puentes de AMI izquierda a arteria descendente anterior (DA), venoso a arteria circunfleja (AC) y venoso a arteria coronaria derecha (CD). Ingresó por cuadro de isquemia aguda de miembro inferior derecho y se confirmó con ecodoppler (ED) aortoilíaco y de miembros inferiores oclusión de arteria femoral superficial proximal derecha y estenosis significativa de arteria ilíaca común derecha. El ecocardiograma transtorácico (ETT) mostró deterioro moderado de la función sistólica del ventrículo izquierdo (FSVI) e insuficiencia mitral (IM) leve. Se realizó angioplastia de arteria ilíaca derecha con colocación de stent metálico y puente femoropoplíteo ipsolateral con resolución del cuadro.

En el posoperatorio inmediato presentó edema agudo de pulmón con angina de pecho y dolor de hombro izquierdo. La exploración física identificó presión arterial (PA) de 140/90 en el brazo derecho y de 120/70 en el izquierdo, taquicardia regular, soplo holosistólico en foco mitral y estertores crepitantes en ambos campos pulmonares. El electrocardiograma mostró taquicardia sinusal a $130 \mathrm{lpm}$ con infradesnivel del ST de V2 a V5. El estudio de laboratorio registró elevación de troponina T ultrasensible (102 ng/dl). El ETT delineó deterioro grave de la FSVI, fracción de eyección (FE) de 30\% con empeoramiento de la motilidad de segmentos anteriores e IM grave funcional con ausencia de signos de disección aórtica. También se solicitó ecodoppler de vasos supraaórticos (EDVSA), un estudio que incluye el análisis de las arterias subclavias, vertebrales y sistema carotídeo extracraneal, que identificó un flujo 


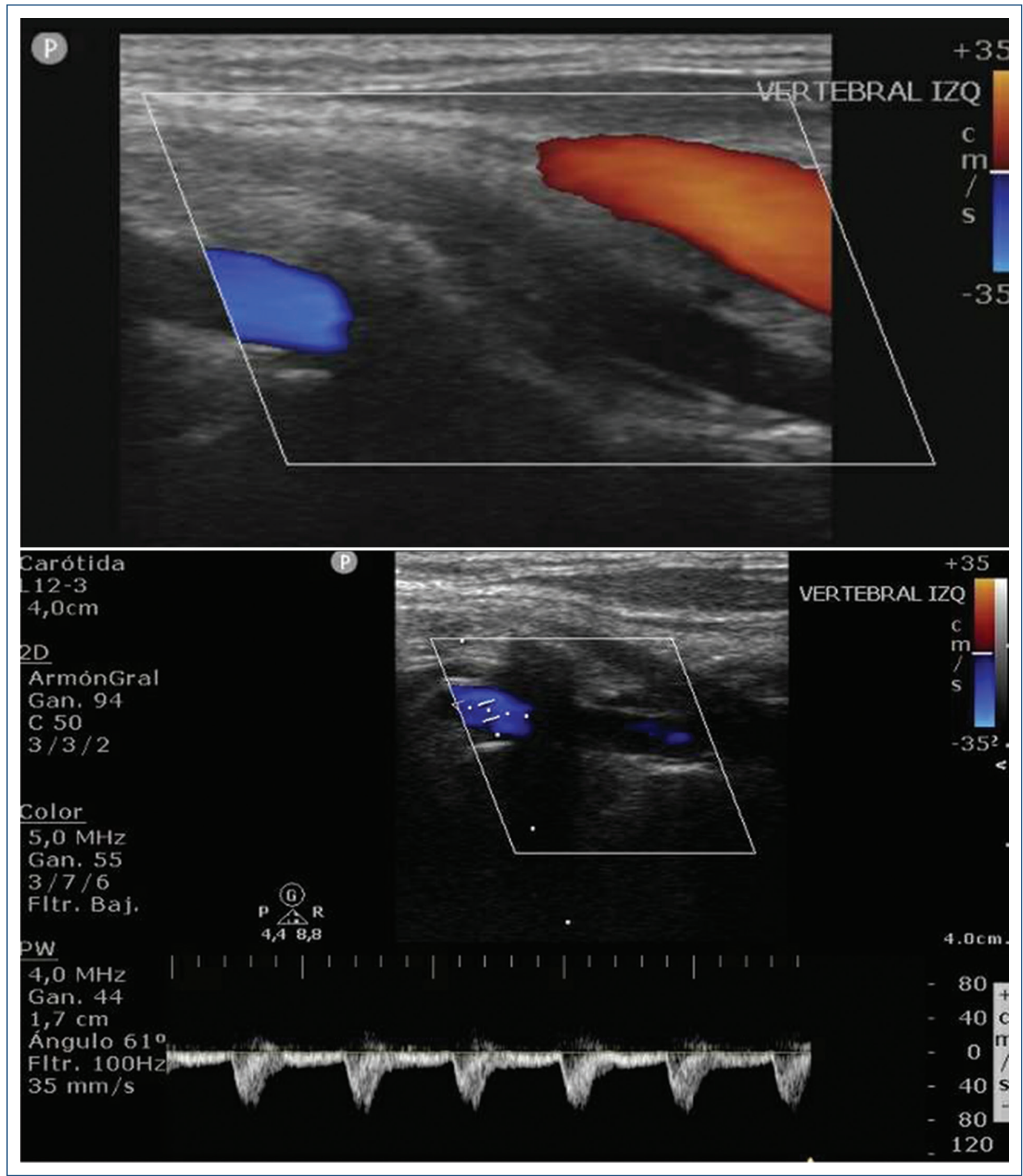

Figura 1. Ecodoppler de vasos supraaórticos. En la imagen superior con Doppler a color se observa en rojo el flujo carotídeo anterógrado y en azul el flujo vertebral retrógrado. En la imagen inferior se muestra el flujo reverso completo en la arteria vertebral izquierda con Doppler pulsado.

reverso completo en la arteria vertebral izquierda $(\mathrm{AVI})$ (Fig. 1). Ante estos hallazgos y con sospecha de síndrome de robo subclavio coronario (SRSC) se efectuó cineangiocoronariografía (CCG) y cateterismo de ambas AS. En la CCG se observó compromiso grave del tercio proximal de $D A, A C$ y $C D$, puente venoso a CD permeable y oclusión de puente venoso a AC. Asimismo, se reconoció una oclusión crónica de la AS izquierda proximal y se confirmó mediante el cateterismo de la AS derecha recanalización de la AMI 


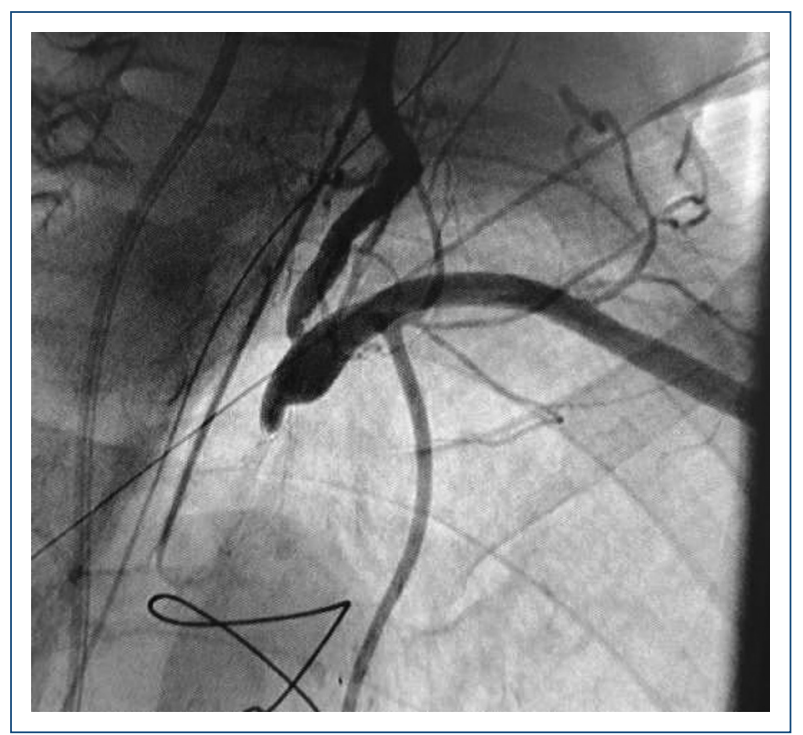

Figura 2. Angiografía digital. Se observa oclusión proximal de arteria subclavia izquierda con recanalización distal desde la arteria vertebral homolateral y puente mamario interno sin lesiones de consideración angiográfica.

desde la $\mathrm{AVI}$, sin evidencian de lesiones del puente mamario (Fig. 2). Se realizó un intento fallido de angioplastia y al final se decidió la revascularización quirúrgica con puente carótido-subclavio izquierdo. Evolucionó sin presentar nuevos episodios de angina. Se repitió el ETT que observó mejoría de la FE (45\%) y disminución de la IM, además de confirmar el EDVSA en el que se identificó flujo vertebral anterógrado conservado.

\section{Discusión}

Se ha comunicado que, en los pacientes con enfermedad vascular periférica, la presencia de algún grado de estenosis subclavia es del $7 \%$ y aumenta a $11.8 \%$ en pacientes que además sufren enfermedad coronaria. Dentro de este último grupo se ha observado que entre $0.8 \%$ y $6.2 \%$ desarrollarán SRSC ${ }^{2}$. A pesar de estos datos, las últimas guías de revascularización miocárdica de la Sociedad Europea de Cardiología del año 2018 no prevén esta entidad como una posible complicación de la revascularización con AMl y por tanto no proponen ningún tipo de cribado para esta alteración antes o después de la intervención ${ }^{2,3}$.

El método más simple para detectar este síndrome es la medición de PA en ambos brazos: una diferencia $>15 \mathrm{mmHg}$ es suficiente para mantener una alta sospecha de estenosis de la AS, aunque podría no existir dicha diferencia en los pacientes con enfermedad bilateral ${ }^{4}$. Desde la descripción original de Harjola de síndrome en 1974 se ha propuesto la búsqueda sistemática de esta anomalía en pacientes elegibles para ORM mediante el uso de angiografía digital; ésta es la norma de referencia para el diagnóstico ${ }^{5}$. Otros métodos como la ultrasonografía, la angiotomografía y la angiorresonancia son útiles y tienen un alto valor predictivo negativo y positivo².

Mediante el estudio del flujo vertebral, el EDVSA es capaz de detectar de forma indirecta la estenosis de la AS. El flujo reverso vertebral completo en reposo o con maniobras de provocación es indicativo de estenosis crítica u oclusión de la $\mathrm{AS}^{6}$. Dado que esta técnica es de fácil acceso, no invasiva y de bajo costo, resulta muy útil en la valoración de pacientes con angina y antecedente de ORM, así como también en el estudio de pacientes aptos para ORM con puente de AMI.

\section{Conclusión}

EI SRSC es una entidad poco frecuente pero con elevada morbilidad que puede complicar a los pacientes con antecedente de ORM. Este caso clínico remarca la utilidad de la clínica y del EDVSA para la aproximación diagnóstica del síndrome en estos pacientes.

\section{Financiamiento}

Ninguno.

\section{Conflicto de intereses}

Los autores declaran no tener conflicto de intereses.

\section{Responsabilidades éticas}

Protección de personas y animales. Los autores declaran que para esta investigación no se han realizado experimentos en seres humanos ni en animales.

Confidencialidad de los datos. Los autores declaran que han seguido los protocolos de su centro de trabajo sobre la publicación de datos de pacientes.

Derecho a la privacidad y consentimiento informado. Los autores han obtenido el consentimiento informado de los pacientes o sujetos referidos en el artículo. Este documento obra en poder del autor de correspondencia. 
Arch Cardiol Mex. 2022;92(1)

\section{Bibliografía}

1. Olsen C, Dunton R, Maggs $P$, Lahey S. Review of coronary-subclavian steal following internal mammary artery-coronary artery by-pass surgery. Ann Thorac Surg. 1988;46:675-678.

2. Cua B, Mamdani N, Halpin D, Jhamnani S, Jayasuriya S, Mena-Hurtado C. Review of coronary subclavian steal syndrome. Journal of Cardiology. 2017;70:432-437.
3. Neumann FJ. 2018 ESC/EACTS Guidelines on Myocardial Revascularization Eurolntervention. 2019 Feb 20;14(14):1435-1534. doi: 10.4244/EIJY19M01_01.

4. Osborn LA, Vernon SM, Reynolds B, Timm TC, Allen K. Screening for subclavian artery stenosis in patients who are candidates for coronary bypass surgery. Catheter Cardiovasc Interv. 2002;56:162-5.

5. Harjola PT, Valle M. The importance of aortic arch or subclavian angiography before coronary reconstruction. Chest. 1974:66:436-8.

6. Shäberle W. Ultrasonography in vascular diagnosis. $2^{\text {nd }}$ ed. Springer 2011:331-332. 